ELECTRONIC RESEARCH ANNOUNCEMENTS OF THE AMERICAN MATHEMATICAL SOCIETY

Volume 5, Pages 1-10 (January 25, 1999)

S $1079-6762(99) 00054-2$

\title{
WAVELETS ON GENERAL LATTICES, ASSOCIATED WITH GENERAL EXPANDING MAPS OF $\mathbf{R}^{n}$
}

\author{
A. CALOGERO
}

(Communicated by Stuart Antman)

\begin{abstract}
In the context of a general lattice $\Gamma$ in $\mathbf{R}^{n}$ and a strictly expanding map $M$ which preserves the lattice, we characterize all the wavelet families, all the MSF wavelets, all the multiwavelets associated with a Multiresolution Analysis (MRA) of multiplicity $d \geq 1$, and all the scaling functions. Moreover, we give several examples: in particular, we construct a single, MRA and $C^{\infty}\left(\mathbf{R}^{n}\right)$ wavelet, which is nonseparable and with compactly supported Fourier transform.
\end{abstract}

\section{INTRODUCTION}

An orthonormal wavelet is a function $\psi \in L^{2}(\mathbf{R})$ such that the set

$$
\left\{\psi_{j, k} \equiv 2^{j / 2} \psi\left(2^{j} x-k\right): j, k \in \mathbf{Z}\right\}
$$

is an orthonormal basis for $L^{2}(\mathbf{R})$. A complete characterization of these wavelets is given by the equations

$$
\begin{array}{ll}
\sum_{j \in \mathbf{Z}}\left|\widehat{\psi}\left(2^{j} \xi\right)\right|^{2}=1 & \text { a.e. } \xi \in \mathbf{R}, \\
\text { b) } \sum_{j=0}^{\infty} \widehat{\psi}\left(2^{j} \xi\right) \overline{\widehat{\psi}\left(2^{j}(\xi+2 k \pi)\right)}=0 & \text { a.e. } \xi \in \mathbf{R}, k \in 2 \mathbf{Z}+1,
\end{array}
$$

together with the assumption $\|\psi\|_{2} \geq 1$. These two equations have been known since the beginning of the theory of wavelets (see [L1] and [L2]), but the proof of this characterization did not appear in the literature until recently (see [Gr], [W], [H-W-W3], [Ga]). A much more direct argument gives us two equations that characterize the orthonormality of the system $\left\{\psi_{j, k}\right\}, j, k \in \mathbf{Z}$ :

$$
\begin{array}{ll}
\sum_{k \in \mathbf{Z}}|\widehat{\psi}(\xi+2 k \pi)|^{2}=1 & \text { a.e. } \xi \in \mathbf{R}, \\
\text { b) } \sum_{k \in \mathbf{Z}} \widehat{\psi}\left(2^{j}(\xi+2 k \pi)\right) \overline{\widehat{\psi}(\xi+2 k \pi)}=0 & \text { a.e. } \xi \in \mathbf{R}, \text { if } j>0 .
\end{array}
$$

These two equations clearly must be a consequence of $(I)$ (see [H-W], Chapter 7).

These four equations are most useful for the construction of very general examples of wavelets. An immediate consequence is that $|\widehat{\psi}(\xi)| \leq 1$ a.e. when $\psi$ satisfy

Received by the editors July 13, 1998.

1991 Mathematics Subject Classification. Primary 42C15.

Key words and phrases. Wavelets, multiresolution analysis (MRA), general lattices in $\mathbf{R}^{n}$, MSF wavelets, multiwavelets. 
$(I)$ a), or $(I I)$ b). Integrating the series in $(I I)$ a) on $[0,2 \pi)$ it then follows that meas $(\operatorname{supp} \widehat{\psi}) \geq 2 \pi$ and we have equality if and only if $|\widehat{\psi}|=\chi_{K}$, with $K$ a measurable subset of $\mathbf{R}$ of measure $|K|=2 \pi$. Wavelets of this last described form are known as Minimally Supported Frequency (MSF) wavelets. It is an easy exercise that the MSF wavelets are characterized by the equations $(I)$ a) and $(I I)$ a) (see $[\mathrm{H}-\mathrm{W}])$.

Another important way for constructing wavelets is the use of the Multiresolution Analysis (MRA) method which yields what is known as the MRA wavelets. This type of wavelets has been completely characterized by Gripenberg and Wang ([Gr], $[\mathrm{W}])$ in the following way: the wavelet $\psi$ is an MRA wavelet if and only if

$$
D_{\psi}(\xi)=\sum_{j=1}^{\infty} \sum_{k \in \mathbf{Z}}\left|\widehat{\psi}\left(2^{j}(\xi+2 k \pi)\right)\right|^{2}=1 \quad \text { a.e. } \xi \in \mathbf{R} .
$$

These notions extend to $\mathbf{R}^{n}, n>1$. Certain needed changes are obvious (we translate by lattice points and the preservation of the norm is affected by multiplying $\psi$ in (1) by $2^{j n / 2}$ instead of $2^{j / 2}$ ). Other changes, however, are more crucial and of a different character. There is a natural extension of the notion of an MRA involving one scaling function. This leads to the same characterization (2) in the $n$-dimensional case. However, an easy computation shows the necessity of introducing $2^{n}-1$ functions $\psi^{1}, \psi^{2}, \ldots, \psi^{L}, L=2^{n}-1$, that generate the wavelets basis. Moreover, another way in which more than one generator appears is by considering an MRA given by more than one scaling function. Taking into account these features one can, again, characterize all these wavelets by two equations $[\mathrm{F}-\mathrm{G}-\mathrm{W}-\mathrm{W}]$ and the basic structure of the 1-dimensional proof applies. This general approach also includes singly generated wavelets (which, of course, cannot be MRA wavelets).

The principal purpose of our work is to show that all this can be extended to the case where the "dilations" are affected by using more general matrices $M$ (not just $M=2 I_{n}$ ) and the translations involve a general lattice. In fact, we obtain a theorem that characterizes these more general wavelets and, also, one that characterizes MRA wavelets and scaling functions. Let us be explicit: in the paper [Ca1] that is to appear in the Journal of Geometric Analysis we obtain a generalization of $(I)$ a), b). The characterization of MSF wavelets offers no surprises. We do this explicitly in the second section. Others have considered these dilations and lattices in the general MRA case: Gröchenig, Madych and Strichartz (we will refer to them below). In the third section we give a characterization of the appropriate Multiresolution Analyses and associated scaling functions (that extend the 1-dimensional result in $[\mathrm{H}-\mathrm{W}]$, Chapter 7). In this connection see also [Ma].

An interesting feature of these general wavelets is that for certain dilations we can construct singly generated MRA wavelets. These and other observations will be made in the fourth section where we list several examples of these more general wavelets. We include a specific method for "smoothing" an MSF wavelet of the plane that, almost certainly, is applicable in much more general situations.

Finally, we wish to thank Guido Weiss for his invaluable advice and help.

\section{Characterization of Wavelet families and MSF Wavelets}

In the mathematical literature there are several papers devoted to the study of wavelets and multiresolution analyses from the point of view of general lattices. The papers $[\mathrm{Ma}]$ and $[\mathrm{S} 1]$ contain detailed illustrations of multiresolution analyses, 
generated by lattice translates of the scaling function and their dilations. Moreover, they show how to construct orthonormal wavelets bases in this context. In particular, Gröchenig and Madych [G-M], and Strichartz [S2], starting from self-affine and self-similar tilings of $\mathbf{R}^{n}$, construct multiresolution analyses and wavelet bases with the translation lattice and scaling naturally associated with the tiling.

We consider a lattice $\Gamma$ of $\mathbf{R}^{n}$ as a discrete additive subgroup of $\mathbf{R}^{n}$ given by the integral linear combinations of $n$ independent vectors. In other words, $\Gamma=A \mathbf{Z}^{n}$ where $A$ is a $n \times n$ real matrix with $\operatorname{det} A>0$. Moreover we $\operatorname{denote}$ by $\mathcal{C}$ the fundamental domain of $\Gamma$ which is the set $\mathcal{C}=A[-1 / 2,+1 / 2)^{n}$.

We define the dual lattice $\Gamma^{*}$ as the set of points $\gamma^{*} \in \mathbf{R}^{n}$ such that $\left\langle\gamma, \gamma^{*}\right\rangle \in 2 \pi \mathbf{Z}$ for all $\gamma \in \Gamma$. It is immediate to see that $\Gamma^{*}=2 \pi\left(A^{*}\right)^{-1} \mathbf{Z}^{n}$. Note that for $n=1$ and $\Gamma=\mathbf{Z}$, we simply have $\Gamma^{*}=2 \pi \mathbf{Z}$.

We say that a linear trasformation $M: \mathbf{R}^{n} \rightarrow \mathbf{R}^{n}$ is an acceptable dilation for $\Gamma$ $[\mathrm{Ma}]$ if it satisfies the following conditions:

(1) $M$ is strictly expanding, i.e. all the eigenvalues $\lambda$ of $M$ satisfy $|\lambda|>1$,

(2) $M$ preserves the lattice $\Gamma$, i.e. $M \Gamma \subseteq \Gamma$.

It is easy to see that $m=|\operatorname{det} M|$ is an integer $\geq 2$. We denote by $M^{*}$ the dual map of $M$.

A family of functions $\Psi=\left\{\psi^{1}, \psi^{2}, \ldots, \psi^{L}\right\} \subset L^{2}\left(\mathbf{R}^{n}\right)$ is a family of orthonormal wavelets when

$$
\left\{\psi_{j, \gamma}^{l}: \gamma \in \Gamma, j \in \mathbf{Z}, l=1, \ldots, L\right\}
$$

is an orthonormal basis for $L^{2}\left(\mathbf{R}^{n}\right)$, where $\psi_{j, \gamma}^{l}=m^{j / 2} \psi^{l}\left(M^{j} x-\gamma\right)$.

In [Ca1] we have characterized these wavelet families as follows:

Theorem 1. Suppose $\Psi=\left\{\psi^{1}, \psi^{2}, \ldots, \psi^{L}\right\} \subset L^{2}\left(\mathbf{R}^{n}\right)$, with $\left\|\psi^{l}\right\|_{2} \geq 1$ for $1 \leq$ $l \leq L$. Then $\Psi$ is a family of orthonormal wavelets if and only if the functions $\psi^{1}, \psi^{2}, \ldots, \psi^{L}$ satisfy the following two equations:
a) $\sum_{l=1}^{L} \sum_{j \in \mathbf{Z}}\left|\widehat{\psi}^{l}\left(M^{* j} \xi\right)\right|^{2}=\operatorname{det} A$
a.e. $\xi \in \mathbf{R}^{n}$,
b) $\sum_{l=1}^{L} \sum_{j=0}^{\infty} \widehat{\psi}^{l}\left(M^{* j} \xi\right) \overline{\widehat{\psi}^{l}\left(M^{* j}\left(\xi+\gamma^{*}\right)\right)}=0 \quad$ a.e. $\xi \in \mathbf{R}^{n}, \gamma^{*} \in \Gamma^{*} \backslash M^{*} \Gamma^{*}$.

This characterization follows the strategy of the proof of Frazier, Garrigós, Wang and Weiss in the classical case. However, there are several differences. For example, in the general case $M$ will not be isotropic, since there may be different eigenvalues. Moreover, $M$ may involve a rotation, so that a neighborhood $U$ of the origin in general will not be contained in $M U$. Thus, we have to take into account several features of lattices which do not show up in the classical case. In some parts of the proof this results in more technical complications; but, in other parts, we have to prove additional results in order to obtain finer estimates.

We recall (see $[\mathrm{H}-\mathrm{W}-\mathrm{W} 1]$ and $[\mathrm{H}-\mathrm{W}-\mathrm{W} 2]$ ) that a Minimally Supported Frequency (MSF) wavelet is a wavelet in $L^{2}\left(\mathbf{R}^{n}\right)$ such that the measure of $\operatorname{supp}(\widehat{\psi})$ is minimal. Here, if $\psi$ is an MSF wavelet, then $|\widehat{\psi}|=\sqrt{\operatorname{det} A} \cdot \chi_{T}$ for some measurable set $T$ in $\mathbf{R}^{n}$ with meas $(T)=(2 \pi)^{n}(\operatorname{det} A)^{-1}$. Fang and Wang present a thorough study of MSF wavelets in $L^{2}(\mathbf{R})$ in the classical context. Dai, Larson and Speegle [D-L-S1] proved that for $\Gamma=\mathbf{Z}^{n}$ and every acceptable map there exists an MSF wavelet. In other words, there always exists a measurable set $K \subset \mathbf{R}^{n}$ such that 
the single function $\psi$, defined by $\widehat{\psi}=$ const $\cdot \chi_{K}$, is an orthonormal wavelet for $L^{2}\left(\mathbf{R}^{n}\right)$. This type of set $K$ is called a wavelet set. Soardi and Weiland [S-W] provided explicit examples of such $K$, for $\Gamma=\mathbf{Z}^{n}$ and $M=2 I$.

The characterization of all the MSF wavelets can be given in terms of the following definition.

Definition 1. Let us define a partition of a measurable set $K \subseteq \mathbf{R}^{n}$ as a countable collection $\left\{K_{n}\right\}$ of measurable subsets of $K$, such that $K_{n} \cap K_{m}$ has measure zero if $n \neq m$ and such that $\bigcup_{n} K_{n}=K$ up to a set of measure zero.

The characterization of all the MSF follows directly from the equations $\left(I^{\prime}\right)$.

Theorem 2. Let $\psi$ be a function such that $|\widehat{\psi}|=\alpha \cdot \chi_{T}$, for some measurable set $T$ and some positive constant $\alpha$. Then $\psi$ is an MSF wavelet if and only if the following three conditions are satisfied:

(a) $\quad \alpha^{2}=\operatorname{det} A$;

(b) $\quad\left\{M^{* j} T: j \in \mathbf{Z}\right\}$ is a partition of $\mathbf{R}^{n}$;

(c) $\left\{T+\gamma^{*}: \gamma^{*} \in \Gamma^{*}\right\}$ is a partition of $\mathbf{R}^{n}$.

Another characterization of MSF wavelets can be found in $[\mathrm{B}-\mathrm{M}-\mathrm{M}]$. The problem which naturaly arises is how to construct the support $T$ of the Fourier transform of an MSF wavelet. In [Ca1] we give a general and constructive method to do this and apply this method to produce some examples which, in particular, involve the quincunx lattice.

\section{Characterization of multiwavelet FAmilies AND SCAling FUnCtions}

In the wavelet literature and, in particular, in the study of digital signal processing there is an increasing interest in wavelets associated with an MRA of multiplicity $d>1$, the so called multiwavelets (see for example $[\mathrm{H}],[\mathrm{Ma}]$, [D-S]). Recent papers show how to convert a single input stream to multi-inputs which give samples of the data at half-intervals. An example is the Geronimo-Hardin-Massopust multiwavelets $[\mathrm{G}-\mathrm{H}-\mathrm{M}]$ which give experimental results in compression.

Let us recall the defition of Multiresolution Analyses (MRA) of multiplicity $d$. We say that a sequence $\left\{V_{j}\right\}, j \in \mathbf{Z}$, of closed subspaces of $L^{2}\left(\mathbf{R}^{n}\right)$ is a multiresolution analysis of multiplicity $d$ if the following four conditions are satisfied:

(i) $\bigcup_{j=-\infty}^{+\infty} V_{j}$ is dense in $L^{2}\left(\mathbf{R}^{n}\right)$;

(ii) $V_{j-1} \subseteq V_{j}$, for every $j \in \mathbf{Z}$;

(iii) for every $f \in L^{2}\left(\mathbf{R}^{n}\right)$ and $j \in \mathbf{Z}$ we have $f(x) \in V_{j} \Longleftrightarrow f\left(M^{-j} x\right) \in V_{0}$;

(iv) there exist $d$ functions $\varphi_{1}, \varphi_{2}, \ldots, \varphi_{d}$ in $V_{0}$ such that $\left\{\varphi_{h}(x-\gamma) ; \gamma \in \Gamma, h=\right.$ $1, \ldots, d\}$ is an orthonormal basis for $V_{0}$.

The functions $\varphi_{1}, \varphi_{2}, \ldots, \varphi_{d}$ are called the scaling functions of the MRA. Furthermore the wavelets associated with an MRA of multiplicity $d$ are called multiwavelets.

In the study of MRA wavelet families, we note an interesting difference between the dyadic map and some general acceptable maps of $\mathbf{R}^{n}$. In the first case one cannot have a single MRA wavelet if $n>1$, while for such $M$ it suffices to consider the case $m=|\operatorname{det} M|=2$ to construct such a single $\psi$, as shown in section 4 .

Theorem 3. Let $\Psi=\left\{\psi^{1}, \psi^{2}, \ldots, \psi^{(m-1) d}\right\} \subset L^{2}\left(\mathbf{R}^{n}\right)$ be a wavelet family associated with the lattice $\Gamma$ and the acceptable map $M . \Psi$ is an MRA wavelet family of 
multiplicity $d$ if and only if

$$
D_{\Psi}(\xi)=\sum_{l=1}^{(m-1) d} \sum_{j=1}^{\infty} \sum_{\gamma^{*} \in \Gamma^{*}}\left|\widehat{\psi}^{l}\left(M^{* j}\left(\xi+\gamma^{*}\right)\right)\right|^{2}=d \cdot \operatorname{det} A \quad \text { a.e. } \xi \in \mathbf{R}^{n} .
$$

A proof of this result is given in $[\mathrm{C}-\mathrm{G}]$ as a consequence of a characterization of wavelet families arising from biorthogonal MRA's of multiplicity $d$. Given the result of Theorem 3, it is of some interest to know which functions can be scaling functions of an MRA. Let us mention existing results on this subject.

The characterization of single scaling functions was previously known in some particular cases: for $n=1$ and so called localized scaling functions (see e.g. [K-L], $[\mathrm{Co}]$ ); for scaling functions which are characteristic functions of a prototile of a selfsimilar tiling of $\mathbf{R}^{n}[\mathrm{G}-\mathrm{M}]$; for certain scaling functions constructed by means of tilings of $\mathbf{R}^{n}$ [S1]. The book [H-W] (see also [H-W-W3]) contains a characterization of all scaling functions in the case $n=1$. Madych, in this context of general lattices and acceptable dilations, characterizes the single functions $\varphi$ which generate an MRA and those whose scaling functions are characteristic functions are completely described.

In the case $d>1$, Geronimo, Hardin and Massopust [G-H-M] give necessary and sufficient conditions for certain functions, constructed using fractal functions, to form an MRA of $L^{2}(\mathbf{R})$. Hervé $[\mathrm{H}]$ studied the scaling filters which yield an MRA of multiplicity $d>1$, while De Michele and Soardi [D-S] studied the basic properties of MRA of multiplicity $d$.

We can prove the following general characterization (see [Ca2]).

Theorem 4. Let $\varphi_{1}, \varphi_{2}, \ldots, \varphi_{d}$ be functions of $L^{2}\left(\mathbf{R}^{n}\right)$ and, for every $j \in \mathbf{Z}$, let

$$
V_{j}=\overline{\operatorname{span}\left\{m^{j / 2} \varphi_{h}\left(M^{j} x-\gamma\right): \gamma \in \Gamma, h=1, \ldots, d\right\}} .
$$

Then the scale of spaces $\left\{V_{j}\right\}_{j \in \mathbf{Z}}$ is an MRA of multiplicity $d$ with scaling functions $\varphi_{1}, \varphi_{2}, \ldots, \varphi_{d}$ if and only if for a.e. $\xi \in \mathbf{R}^{n}$ the following conditions hold:

$$
\begin{gathered}
\sum_{\gamma^{*} \in \Gamma^{*}} \widehat{\varphi}_{h}\left(\xi+\gamma^{*}\right) \overline{\widehat{\varphi}_{k}\left(\xi+\gamma^{*}\right)}=0 \quad \text { for } h, k=1, \ldots, d \text { with } k \neq h \\
\lim _{j \rightarrow+\infty} \sum_{h=1}^{d}\left|\widehat{\varphi}_{h}\left(M^{*-j} \xi\right)\right|^{2}=\operatorname{det} A \\
\widehat{\varphi}_{h}\left(M^{*} \xi\right)=\sum_{k=1}^{d} m_{h k}(\xi) \widehat{\varphi}_{k}(\xi) \quad \text { for } h=1, \ldots, d
\end{gathered}
$$

for some functions $m_{h k}$ of $L^{2}\left(\mathbf{R}^{n} / \Gamma^{*}\right)$.

We recall that in the case $d=1$, the function $m$ which satisfies relation (6) is called a low-pass filter. 


\section{Examples of MSF and MRA WAVELEts, of MUltiwaVelets AND A $C^{\infty}\left(\mathbf{R}^{2}\right)$, NONSEPARABLE WAVELET WITH COMPACTLY SUPPORTED FOURIER TRANSFORM}

In this last section we give some examples of wavelets constructed from the previous characterizations. In particular, we concentrate our attention on the case $\Gamma=\mathbf{Z}^{2}$ and the acceptable map $M(x, y)=(x-y, x+y)$ which was studied in some detail by Cohen and Daubechies [C-D]. For this map $m=|\operatorname{det} M|=2$. Clearly $M^{*}(x, y)=(x+y, y-x)$ and $M^{*-1}(x, y)=((x-y) / 2,(y+x) / 2)$.

In the first example we produce an MRA, MSF wavelet $\psi_{0}$. In the second example, we smooth the wavelet $\psi_{0}$. So we obtain a single $C^{\infty}\left(\mathbf{R}^{2}\right)$, nonseparable wavelet with compactly supported Fourier transform. Using the ideas of the construction of $\psi_{0}$, in the third example, we present two wavelets $\psi_{1}$ and $\psi_{2}$ that are multiwavelets associated with an MRA of multiplicity 2. In the fourth example, we consider a different map and give examples of singly generated wavelts in $\mathbf{R}^{n}$.

Example 1. Let us define $T=M^{*} \mathcal{C}^{*} \backslash \mathcal{C}^{*}$. We recall that $\mathcal{C}^{*}=[-\pi, \pi)^{2}$. We claim that $\widehat{\psi}_{0}=\chi_{T}$ defines a wavelet $\psi_{0}$ associated with a multiresolution analysis of multiplicity 1 . Moreover, $\psi_{0}$ is also an MSF wavelet.

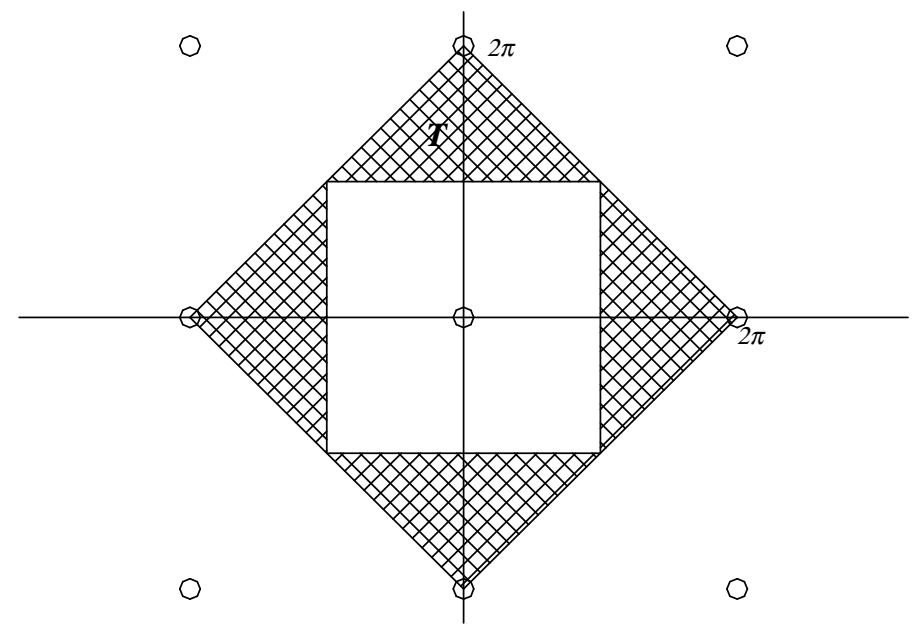

Figure 1. The support $T$ of the Fourier transform of the wavelet $\psi_{0}$.

The proof is an easy application of Theorems 2 and 3. We do not give this proof here, but we indicate how it is possible to construct the wavelet $\psi_{0}$ starting from the scaling function $\varphi_{0}$ of the MRA. We define the scale of spaces $\left\{V_{j}\right\}_{j \in \mathbf{Z}}$ of $L^{2}\left(\mathbf{R}^{n}\right), V_{j}=\overline{\operatorname{span}\left\{2^{j / 2} \varphi_{0}\left(M^{j} x-\gamma\right): \gamma \in \mathbf{Z}^{2}\right\}}$, with the scaling function $\widehat{\varphi}_{0}=\chi_{\mathcal{C}^{*}}$ (see Theorem 4). Moreover an easy computation shows that the low-pass filter is $m_{0}(\xi)=\sum_{\gamma^{*} \in 2 \pi \mathbf{Z}^{2}} \chi_{\left(M^{*-1} \mathcal{C}^{*}\right)}\left(\xi+\gamma^{*}\right)$.

With this fixed map and lattice, we use that a generic scaling function $\varphi$ and the associated low-pass filter $m$ produce the wavelet $\psi$ by

$$
\widehat{\psi}\left(M^{*}(x, y)\right)=\overline{m(x+\pi, y+\pi)} \widehat{\varphi}(x, y) e^{i(x+y) / 2} .
$$

We recall that $e^{i(x+y) / 2}$ is called the phase and $p(x, y)=\overline{m(x+\pi, y+\pi)} e^{i(x+y) / 2}$ is called the high-pass filter. Moreover, we remember (see [D-S]) that $p$ is such that 
the matrix

$$
\left(\begin{array}{cc}
m(x, y) & m(x+\pi, y+\pi) \\
p(x, y) & p(x+\pi, y+\pi)
\end{array}\right)
$$

is unitary, a.e. This implies the so called filter relation,

$$
|m(x, y)|^{2}+|m(x+\pi, y+\pi)|^{2}=1 .
$$

Returning to our example, since the wavelet $\psi_{0}$ is MSF, we can choose an arbitrary phase in (7) and it is an easy exercise to obtain $\psi_{0}$ from $\varphi_{0}$. Now the question is the following: in order to construct a $C^{\infty}\left(\mathbf{R}^{2}\right)$ wavelet, is it possible to smooth the previous wavelet $\psi_{0}$ ? The next example gives us a positive answer.

Example 2. In order to answer the previous question, we do not smooth directly the wavelet $\psi_{0}$, but we smooth the filter $m_{0}$. For every fixed $\epsilon$, with $0<\epsilon \leq \pi$, we construct two low-pass filters, $\widetilde{m_{\epsilon}}$ and $m_{\epsilon}$. We will see that the construction of $\widetilde{m_{\epsilon}}$ is very easy but also that this function belongs only to $\operatorname{Lip}_{1}\left(\mathbf{R}^{2}\right)$. However, the idea underlying the construction of $\widetilde{m_{\epsilon}}$ allows us to produce a more complicated function $m_{\epsilon} \in C^{\infty}\left(\mathbf{R}^{2}\right)$.

These constructions are similar to the one that produces the Lemarié-Meyer wavelet from the Shannon wavelet. We begin by defining a $C^{\infty}(\mathbf{R})$ function $\theta$ with the following properties:

$$
\begin{aligned}
& 0 \leq \theta(t) \leq 1 \text { and } \quad \theta(t)=1-\theta(1-t) \quad \text { for every } t \in \mathbf{R}, \\
& \theta(t)=0 \quad \text { if } t \leq 0, \quad \theta(t)=1 \quad \text { if } t \geq 1 .
\end{aligned}
$$

Moreover let us define the function $\nu(t)=\cos \left(\frac{\pi}{2} \theta(t)\right)$. Clearly $\nu \in C^{\infty}(\mathbf{R})$ and $\nu^{2}(t)+\nu^{2}(1-t)=1$. Starting from this function $\nu$, we construct the low-pass filters.

Let us fix $\epsilon$, with $0<\epsilon \leq \pi$. For every $(x, y) \in[-\pi, \pi]^{2}$ we set

$$
\widetilde{m_{\epsilon}}(x, y)=\nu[(|x|+|y|-\pi+\epsilon) /(2 \epsilon)] .
$$

Next, we extend periodically the definition of $\widetilde{m_{\epsilon}}$ so that, for every $\gamma^{*} \in 2 \pi \mathbf{Z}^{2} \backslash\{0\}$ and $\xi \in[-\pi, \pi]^{2}$, we have $\widetilde{m_{\epsilon}}\left(\xi+\gamma^{*}\right)=\widetilde{m_{\epsilon}}(\xi)$. Clearly, $\widetilde{m_{\epsilon}} \in L^{2}\left(\mathbf{R}^{2} / \Gamma^{*}\right)$ and belongs to $\operatorname{Lip}_{1}\left(\mathbf{R}^{2}\right)$. It is easy to verify that $\widetilde{m_{\epsilon}}$ is a low-pass filter.

Let us construct the second low-pass filter $m_{\epsilon}$. For every $(x, y) \in \mathbf{R}^{2}$, we set

$$
\begin{aligned}
n_{\epsilon}(x, y)= & \nu[(x+y-\pi+\epsilon) /(2 \epsilon)] \nu[(x-y-\pi+\epsilon) /(2 \epsilon)] \\
\cdot & \nu[(-x+y-\pi+\epsilon) /(2 \epsilon)] \nu[(-x-y-\pi+\epsilon) /(2 \epsilon)] .
\end{aligned}
$$

Since $\operatorname{supp}\left(n_{\epsilon}\right) \subseteq[-\pi-\epsilon, \pi+\epsilon]^{2}$, we define the function $N_{\epsilon}$ by periodizing $n_{\epsilon}$ :

$$
N_{\epsilon}(\xi)=\sum_{\gamma^{*} \in 2 \pi \mathbf{Z}^{2}} n_{\epsilon}\left(\xi+\gamma^{*}\right)
$$

Clearly, $N_{\epsilon} \in L^{2}\left(\mathbf{R}^{2} / \Gamma^{*}\right) \cap C^{\infty}\left(\mathbf{R}^{2}\right)$, but the filter relation is not satisfied. Since $N_{\epsilon}^{2}(x, y)+N_{\epsilon}^{2}(x+\pi, y+\pi)>0$, we can prove that the function

$$
m_{\epsilon}(x, y)=\frac{N_{\epsilon}(x, y)}{\sqrt{N_{\epsilon}^{2}(x, y)+N_{\epsilon}^{2}(x+\pi, y+\pi)}}, \quad \text { for every }(x, y) \in \mathbf{R}^{2},
$$

is a low-pass filter belonging to $C^{\infty}\left(\mathbf{R}^{2}\right)$. We remark that $\operatorname{supp}\left(\widetilde{m_{\epsilon}}\right)=\operatorname{supp}\left(m_{\epsilon}\right)$. 


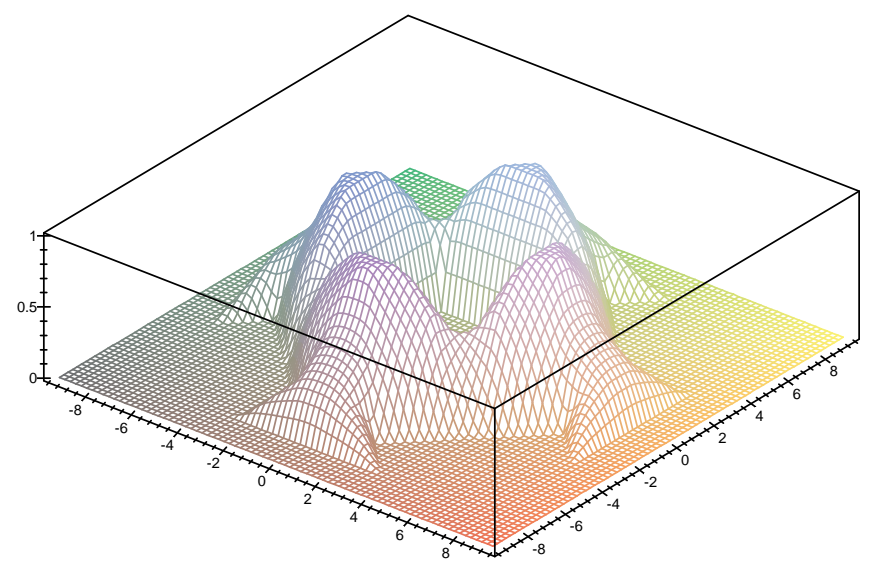

Figure 2. The function $\left|\widehat{\psi}_{\epsilon}\right|$, with $\epsilon=2 / 5$.

Theorem 5. For every fixed $\epsilon$, with $0<\epsilon \leq \pi / 5$, the function $\psi_{\epsilon}$ defined by

$$
\widehat{\psi_{\epsilon}}(x, y)=\prod_{j=2}^{5} m_{\epsilon}\left(M^{*-j}(x, y)\right) m_{\epsilon}\left(M^{*-1}(x, y)+(\pi, \pi)\right) e^{i x / 2},
$$

is a wavelet of $L^{2}\left(\mathbf{R}^{2}\right)$ with lattice $\Gamma=\mathbf{Z}^{2}$ and acceptable map $M$. This wavelet is MRA and belongs to $C^{\infty}\left(\mathbf{R}^{2}\right)$. Moreover, $\psi_{\epsilon}$ is nonseparable and with compactly supported Fourier transform.

The proof of Theorem 5 uses (7) and the relation

$$
\widehat{\varphi}_{\epsilon}(\xi)=\prod_{j=1}^{\infty} m_{\epsilon}\left(M^{*-j} \xi\right)
$$

where $\varphi_{\epsilon}$ is the scaling function of the associated MRA.

We remark that the assumption on $\epsilon$ guarantees that the set

$$
\operatorname{supp}\left(\widehat{\varphi}_{\epsilon}\right)=\bigcap_{j \geq 1}\left(\operatorname{supp}\left(m_{\epsilon}\left(M^{*-j} \cdot\right)\right)\right)
$$

is a compact and connected subset of $[-2 \pi, 2 \pi]^{2}$. Hence, $\operatorname{supp}\left(\widehat{\psi}_{\epsilon}\right)$ is also compact and connected. These properties together with (8) show that $\widehat{\varphi}_{\epsilon}$ is the product of only four factors.

It is possible to show that if we assume $\pi / 5<\epsilon \leq \pi$, then we can construct again an MRA wavelet $\psi_{\epsilon}$ but the scaling function $\varphi_{\epsilon}$ is not a finite product of functions and $\operatorname{supp}\left(\widehat{\varphi}_{\epsilon}\right)$ is unbounded.

Finally, similar results hold with $\widetilde{m_{\epsilon}}$ in place of $m_{\epsilon}$; the corresponding functions $\widehat{\varphi_{\epsilon}}$ and $\widehat{\psi_{\epsilon}}$ are simpler to describe but less regular.

Example 3. Let us define $\Psi=\left\{\psi^{1}, \psi^{2}\right\}$ such that $\widehat{\psi}^{1}=\chi_{T_{1}}$ and $\widehat{\psi}^{2}=\chi_{T_{2}}$.

We claim that $\Psi$ is a wavelet family associated with a multiresolution analyses of multiplicity 2. The proof uses Theorems 1 and 3.

Example 4. In this last example, we want to show that for every integer $n \geq$ 2 , there exists an easy MRA wavelet on the lattice $\Gamma=\mathbf{Z}^{n}$, associated with an acceptable map $M_{n}$ with $\operatorname{det} M_{n}=2$. Let $\widetilde{\psi} \in L^{2}(\mathbf{R})$ be an MRA wavelet on the 

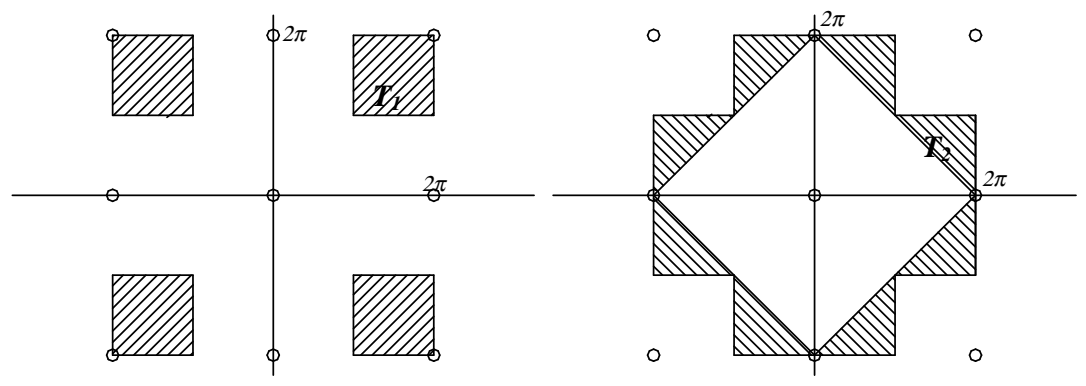

Figure 3 . The supports $T_{1}$ and $T_{2}$ of $\widehat{\psi}^{1}$ and $\widehat{\psi}^{2}$ respectively.

usual lattice $\Gamma=\mathbf{Z}$, associated with the usual dyadic map. Moreover, let $\widetilde{\varphi}$ be the scaling function of the MRA. Let us fix $n \geq 2$ and let us consider the map $M_{n}^{*}\left(x_{1}, x_{2}, \ldots, x_{n}\right)=\left(2 x_{n}, x_{1}, x_{2}, \ldots, x_{n-1}\right)$. We claim that the function $\psi$ such that

$$
\widehat{\psi}\left(x_{1}, x_{2}, \ldots, x_{n}\right)=\widehat{\widetilde{\psi}}\left(x_{1}\right) \widehat{\widetilde{\varphi}}\left(x_{2}\right) \widehat{\widetilde{\varphi}}\left(x_{3}\right) \cdots \widehat{\widetilde{\varphi}}\left(x_{n}\right)
$$

is an MRA wavelet on the lattice $\mathbf{Z}^{n}$, associated with the map $M_{n}$. The proof is an easy exercise.

\section{REFERENCES}

[B-M-M] Bagget W.B., Medina H.A., Merrill K.D.: Generalized multiresolution analyses, and a construction procedure for all wavelet sets in $\mathbf{R}^{n}$, preprint.

[Ca1] Calogero A.: A characterization of wavelets on general lattices, Journal of Geometric Analysis, to appear.

[Ca2] Calogero A.: A characterization of scaling functions of multiresolution analyses on general lattices, preprint (1998).

[Ca3] Calogero A.: Wavelets on general lattices, associated with general expanding maps of $\mathbf{R}^{n}$. Ph. D. Thesis, Universitá di Milano (1998).

[C-G] Calogero A., Garrigós G.: A characterization of wavelet families arising from biorthogonal MRA's of multiplicity d, preprint (1998).

[Co] Cohen A.: Ondelettes et traitement numérique du signal. Research Notes in Mathematics, Masson, Paris (1992). MR 95g:42038

[C-D] Cohen A., Daubechies I.: Nonseparable bidimensional wavelet bases. Revista Matemática Iberoamericana 9, No. 1 (1993). MR 94k:42047

[D-L-S1] Dai X., Larson D., Speegle D.: Wavelet sets in $\mathbf{R}^{n}$, The Journal of Fourier Analysis and Applications 3, 451-456 (1997). MR 98m:42048

[D-L-S2] Dai X., Larson D., Speegle D.: Wavelet sets in $\mathbf{R}^{n}$, II. Contemporary Mathematics 216, 15-40 (1998). CMP 98:11

[D-S] De Michele L., Soardi P.M.: On multiresolution analysis of multiplicity d, Mh. Math 124, 255-272 (1997). MR 98k:42039

[F-G-W-W] Frazier M., Garrigós G., Wang K., Weiss G.: A characteritazion of functions that generate wavelet and related expansion, Journal of Fourier Analysis and Applications, Vol. 3 (special issue), (1997). CMP 98:09

[Ga] Garrigós G.: The characterization of wavelets and related functions and connectivity of $\alpha$-localized wavelets on $\mathbf{R}$, Ph.D. Thesis, Washington University in St. Louis (1998).

[Gr] Gripenberg G.: A necessary and sufficient condition for the existence of a father wavelet, Studia Math. 114 (3) (1995), 207-226. MR 96d:42049

[G-H-M] Geronimo J., Hardin D., Massopust P.R.: Fractal functions and wavelet expansions based on several functions, J. Approx. Theory 78, 373-401 (1994). MR 95h:42033 
[G-M] Gröchenig K., Madych W.R.: Multiresolution analysis, Haar bases, and self-similar tilings of $\mathbf{R}^{n}$, IEEE Trans. Inform. Theory 38, 556-568 (1992). MR 93i:42001

$[\mathrm{H}] \quad$ Hervé L.: Multi-resolution analysis of multiplicity $d$ : applications to dyadic interpolation, Appl. and Comput. Harmonic Anal. 1, 299-315 (1994). MR 97a:42026

[H-W] Hernández E., Weiss G.: A first course on wavelets, CRC Press (1996). MR 97i: 42015

[H-W-W1] Hernández E., Wang X., Weiss G.: Smoothing minimally supported frequency wavelets: part I, Journal of Fourier Analysis and Applications, Vol. 2, No. 4 (1996), 329-340. MR 97h: 42015

[H-W-W2] Hernández E., Wang X., Weiss G.: Smoothing minimally supported frequency (MSF) wavelets: part II, Journal of Fourier Analysis and Applications, Vol. 3, No. 1 (1997), 23-41. MR 98b:42049

[H-W-W3] Hernández E., Wang X., Weiss G.: Characterization of wavelets, scaling function and wavelets associated with multiresolution analysis. Washington University in St. Louis, Preprint (1995).

[K-L] Kahane J. P., Lemarié-Rieusset P.G.: Fourier series and wavelets. Gordon and Breach (1995).

[L1] Lemarié-Rieusset P.G.: Analyse multi-schelles et ondelettes à support compact, Les ondelettes en 1989 (P.G. Lemarié, Ed.), Lecture Notes in Mathematics, 1438, Springer-Verlag (1990), 26-38. MR 92g:42018

[L2] Lemarié-Rieusset P.G.: Ondelettes à localisation exponentielle, J. Math. Pure et Appl. 67 (1988), 227-236.

[Ma] Madych W.R.: Some elementary properties of multiresolution analyses of $L^{2}\left(\mathbf{R}^{n}\right)$, Wavelets: A tutorial in theory and applications (C.K. Chui, Ed.), Academic Press (1992), 259-294. MR 93k:42018

[Me] Meyer Y.: Ondelettes et opérateurs: Ondelettes. Hermann, Paris (1990). MR 93i: 42002

[S-W] Soardi P.M., Weiland D.: Single wavelets in n-dimensions, The Journal of Fourier Analysis and Applications 4, 299-315 (1998). CMP 99:03

[S-N] Strang G., Nguyen T.: Wavelets and filter banks, Wellesley-Cambridge Press (1996). MR 98b:94003

[S1] Strichartz R.S.: Construction of orthonormal wavelets, Wavelets, Mathematics and Applications (J.J: Benedetto and M.W. Frazier, Eds.), CRC Press (1993), 23-50. MR 94i: 42047

[S2] Strichartz R.S.: Wavelets and self-affine tilings, Constr. Approx. 9, 327-346 (1993). MR 94f: 42039

[W] Wang X.: The study of wavelets from the properties of their Fourier trasforms, Ph.D. Thesis, Washington University in St. Louis (1995).

Dipartimento di Matematica, Universitá di Milano, via Saldini 50, 20133 Milano, Italy

E-mail address: Calogero@vmimat.mat.unimi.it 